\title{
Modulation of transcription and characterization of the promoter organization of the autotransporter adhesin heptosyltransferase and the autotransporter adhesin AIDA-I
}

\author{
Inga Benz, Tessa van Alen, Julia Bolte, Mirka E. Wörmann \\ and M. Alexander Schmidt \\ Institut für Infektiologie, Zentrum für Molekularbiologie der Entzündung (ZMBE), Westfälische \\ Wilhelms-Universität Münster, D-48149 Münster, Germany
}

Correspondence

M. Alexander Schmidt

infekt@uni-muenster.de

Received 2 July 2010

Revised 21 December 2009

Accepted 26 December 2009

\begin{abstract}
In Gram-negative bacteria, autotransporter proteins constitute the largest family of secreted proteins, and exhibit many different functions. In recent years, research has largely focused on mechanisms of autotransporter protein translocation, where several alternative models are still being discussed. In contrast, the biogenesis of only a few autotransporters has been studied and, likewise, regulation of expression has received only very limited attention. The glycosylated autotransporter adhesin involved in diffuse adherence (AIDA)-I system consists of the aah gene, encoding a specific autotransporter adhesin heptosyltransferase (AAH), and the aidA gene, encoding the autotransporter protein (AIDA-I). In this study, we investigated the promoter organization and transcription of these two genes using reporter plasmids carrying lac $Z$ transcriptional fusions. The two genes, aah and aid $A$, are transcribed as a bicistronic message. However, aidA is additionally transcribed from its own promoter. There are two distinct start sites for each of the two genes. Interestingly, transcription of both genes is enhanced in $h n s$ and $r f a H$ mutant backgrounds. Furthermore, we addressed the influence of environmental factors and different genetic backgrounds of Escherichia coli K-12 strains on transcription activity. We found that transcription varied considerably in different $E$. coli K-12 laboratory strains and under different growth conditions.
\end{abstract}

\section{INTRODUCTION}

With now more than 800 members, autotransporter proteins constitute the largest family of secreted proteins in Gram-negative bacteria (Dautin \& Bernstein, 2007; Wells et al., 2007). Autotransporters mediate a wide variety of functions, such as adhesion, host cell actin polymerization, and serum resistance. In addition, they may also express or shed enzymes with, e.g. protease, esterase and lipase activities (Dautin \& Bernstein, 2007; Henderson et al., 2004). Plasmid-encoded adhesin involved in diffuse adherence (AIDA)-I was isolated from the clinical Escherichia coli strain 2787 (O126:H27) and mediates the diffuse adherence phenotype of the wild-type bacterium to HeLa cells (Benz \& Schmidt, 1989).

The distribution of AIDA-I is not restricted to human isolates; on the contrary, AIDA-I is more often identified in porcine E. coli isolates, indicating a putative food-borne infection chain. In these animals, the presence of AIDA-I-

Abbreviations: $\mathrm{AAH}$, autotransporter adhesin heptosyltransferase; AIDA, adhesin involved in diffuse adherence; 5'-RACE, rapid amplification of 5' complementary DNA ends. positive strains appears to be associated with F18 fimbriae and oedema disease (Niewerth et al., 2001; Mainil et al., 2002). That AIDA-I augments virulence in intestinal disorders is supported by findings in vivo that show that expression of AIDA-I in porcine strains increases the onset of diarrhoea in weaned piglets (Pritchard et al., 2004).

AIDA-I is encoded by the aidA gene and is synthesized as a $132 \mathrm{kDa}$ pre-pro-protein featuring an unusually long 49 aa signal peptide (Benz \& Schmidt, 1992a), as has also been found in other autotransporters. For IcsA of Shigella flexneri, it has been shown that the protein is cleaved during Sec-dependent transport through the inner membrane (Brandon et al., 2003). In the periplasm, the C-terminal part ('translocator') of the protein is integrated as a $\beta$-barrel into the outer membrane (Suhr et al., 1996). The mechanism of surface translocation of the adhesin ('passenger' domain) involving the translocator domain still remains elusive and various alternative mechanistic models have been proposed (Jain \& Goldberg, 2007). During the translocation process the AIDA pro-protein is cleaved autocatalytically and the mature AIDA-I adhesin remains associated with the outer 
membrane on the bacterial surface. A $119 \mathrm{kDa}$ glycoprotein on the surface of HeLa cells has been identified as a docking protein for the AIDA adhesin (Laarmann \& Schmidt, 2003).

For full adhesive function and/or stability, AIDA-I has to be modified by a second protein encoded directly upstream of the aidA gene (Benz \& Schmidt, 2001). This protein has been shown to function as a protein heptosyltransferase, and is accordingly termed the 'autotransporter adhesin heptosyltransferase' (AAH). AAH catalyses the addition of, on average, 19 heptose residues to the AIDA protein (Benz \& Schmidt, 2001). For this, AAH uses ADP-glyceromanno-heptopyranose as a donor from the lipopolysaccharide synthesis pathway. AAH appears to be the only protein-heptosylating enzyme known thus far. Not surprisingly, the amount of AIDA-I substrate present in the bacterial cell (Benz \& Schmidt, 1992b) by far exceeds the amount of the heptosylating enzyme AAH. How the balance between the two proteins is maintained to ensure the optimal function of the glycosylated AIDA-I adhesin is not known.

Thus far, expression of bacterial autotransporters has been mostly addressed with respect to modulation by environmental conditions (e.g. Henderson et al., 2004). However, the expression of bacterial glycosylated autotransporters such as TibA or AIDA-I has not been addressed in any detail. The need for a better understanding of autotransporter expression is further underlined by the increasing interest in the AIDA-I translocator $\left(\mathrm{AIDA}^{\mathrm{C}}\right.$ ) for the expression of heterologous proteins on the surface of Gram-negative bacteria (Konieczny et al., 2000; Casali et al., 2002; Jose \& Meyer, 2007).

In this study, we present evidence that the two genes constituting the AIDA system are transcribed as a bicistronic mRNA from a regulated promoter upstream of aah. In addition, mRNA is also synthesized from a promoter directly upstream of the aidA gene.

\section{METHODS}

Bacterial strains, plasmids and media. E. coli strains used in this study are listed in Table 1. Plasmid pIB264 (Benz \& Schmidt, 1989) is a pBR322 derivative $\left(A m p^{r}\right)$ and harbours the genes for the expression of the authentic AIDA-I system (aah and aidA). pCB192 (Amp ${ }^{\mathrm{r}}$ ) derived from pBR322 is a promoter test vector and harbours the promoterless genes $l a c Z$ and galK (Schneider \& Beck, 1986). Bacteria were generally grown at $37{ }^{\circ} \mathrm{C}$ in Standard I medium (Merck). For the maintenance of plasmids, the medium was supplemented with $100 \mu \mathrm{g}$ ampicillin $\mathrm{ml}^{-1}$. Salt medium was prepared as described by Bäcklund et al. (2008). The minimal medium was supplemented with $0.02 \%$ thiamine and $0.03 \%$ Bacto casamino acids.

Table 1. E. coli strains employed in this study

\begin{tabular}{|c|c|c|}
\hline Strain & Genotype & Source \\
\hline BW25113 & $\Delta(\operatorname{araD}-a r a \mathrm{~B}) 567 \Delta l a c \mathrm{Z} 4787(:: r r n \mathrm{~B}-4) r p h-1 \Delta(r h a \mathrm{D}-r h a \mathrm{~B}) 568 r r n \mathrm{~B}-4$ hsd $\mathrm{R} 514$ & Datsenko \& Wanner (2000) \\
\hline BW25141 & $\begin{array}{l}\Delta(\text { araD-araB)567 } \Delta l a c Z 4787(:: r r n \mathrm{~B}-4) r p h-1 \Delta(r h a \mathrm{D}-r h a \mathrm{~B}) 568 r r n \mathrm{~B}-4 \text { hsd } \mathrm{R} 514 \\
\Delta(\text { phoB-phoR)580 galU95 } \Delta \text { uidA3::pir+ recA1 endA9(del-ins) }:: \mathrm{FRT}\end{array}$ & Datsenko \& Wanner (2000) \\
\hline C600 & $\mathrm{F}^{-}$thi-1 thr-1 leuB6 lacY1 cyn101 glpR200 e14- supE44 rfbC1 fhuA21 & Appleyard (1954) \\
\hline $\mathrm{DH} 5 \alpha$ & 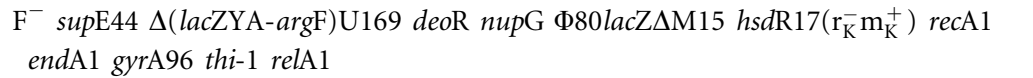 & Hanahan (1983) \\
\hline GM2163 & $\begin{array}{l}\mathrm{F}^{-} \text {araC14 leuB6 fhuA13 lacY1 tsx-78 glnV44 galK2 galT22 mcrA0 dcm-6 hisG4 } \\
\text { rfbC1 rpsL136 dam-13:: Tn9 xylA5 mtl-1 thi-1 mcrB9999 hsdR2 }\end{array}$ & Marinus et al. (1983) \\
\hline JM105 & sup $\mathrm{E} 44$ end 1 thi-1 rpsL $s b c \mathrm{~B} 15$ hsd $\mathrm{R} 4 \Delta$ (lac-pro) $\left[\mathrm{F}^{\prime}\right.$ traD36 pro $\mathrm{AB}^{+}$lacIqZ $\left.\Delta \mathrm{M} 15\right]$ & Yanisch-Perron et al. (1985) \\
\hline JM109 & $\begin{array}{l}\text { recA1 endA1 gyrA96 thi-1 hsd } \mathrm{R} 17\left(\mathrm{r}_{\mathrm{K}}^{-} \mathrm{m}_{\mathrm{K}}^{+}\right) \text {sup } \mathrm{E} 44 \text { relA1 e14- } \Delta(\text { lac-proAB }) \\
{\left[\mathrm{F}^{\prime} \text { traD36 proAB }{ }^{+} \text {lacIqZ } \Delta \mathrm{M} 15\right]}\end{array}$ & Promega \\
\hline MC4100 & $\mathrm{F}^{-} \Delta($ lacZYA-argF)U169 araD139 rpsL150 flhD5301 deoC1 fruA25 relA1 e14- & Powers \& Randall (1995) \\
\hline MG1655 & rph-1 & Jensen $(1993)$ \\
\hline MG1655 $\Delta \mathrm{rfaH}$ & MG1655 $\Delta \mathrm{rfaH}:$ : cat, cat resistance removed by FLP flippase carried by pCP20 & Beloin et al. (2006) \\
\hline ORN103 & $\begin{array}{l}\mathrm{F}^{-} \text {thr-1 leuB6 thi-1 lacY1 xyl-7 ara-13 mtlA2 gal-6 rpsL ton } \mathrm{A} 2 \text { min } \mathrm{A} \text { min } \mathrm{B}-2 \\
\Delta p i l \Delta(\text { lacZYA-argF) } \mathrm{U} 169 \text { recA13 }\end{array}$ & Orndorff et al. (1985) \\
\hline PD145 & MC4100 hns $205:: \operatorname{Tn} 10$ & Dersch et al. (1994) \\
\hline RH90 & MC4100 rpoS359:: Tn 10 & Lange \& Hengge-Aronis (1991) \\
\hline Sure & $\begin{array}{l}\Delta(m r r-h s d \mathrm{RMS}-m c r \mathrm{BC}) \text { endA1 supE44 thi-1 gyrA96 relA1 lac recB recJ sbcC } \\
\left.\text { umuC::Tn5 e14- } u v r \mathrm{C}\left[\mathrm{F}^{\prime} \text { proAB }{ }^{+} \text {laclqZ } \Delta \mathrm{M} 15 \text { Tn } 10 \text { (tet }^{\mathrm{r}}\right)\right]\end{array}$ & Stratagene \\
\hline TB1 & ara $\Delta($ lac-ara $\mathrm{AB}) \operatorname{rpsL} \Phi 80$ lacZ $\Delta \mathrm{M} 15$ hsd $\mathrm{R}$ & Biolabs \\
\hline TG1 & supE hsdD5 thi $\Delta($ lac-proAB $)\left[\mathrm{F}^{\prime}\right.$ traD36 proAB ${ }^{+}$lacIqZ $\left.\Delta \mathrm{M} 15\right]$ & Gibson (1984) \\
\hline top 10 & $\begin{array}{l}\mathrm{F}^{-} \text {mcrA } \Delta(m r r-h s d \mathrm{RMS}-m c r \mathrm{BC}) \text { Ф80lacZ } \Delta \mathrm{M} 15 \text { slacX74 recAl araD139 } \\
\Delta(\text { ara-leu }) 7697 \text { galU galK rpsL endA1 nupG }\end{array}$ & Invitrogen \\
\hline XL1 blue & $\begin{array}{l}\text { recA1 endA1 gyrA96 thi-1 hsd } \mathrm{R} 17\left(\mathrm{r}_{\mathrm{K}}^{-} \mathrm{m}_{\mathrm{K}}^{+}\right) \text {supE44 relA1 lac }\left[\mathrm{F}^{\prime} \text { proAB }{ }^{+}\right. \\
\left.\text {lacIqZ } \Delta \mathrm{M} 15 \operatorname{Tn} 10\left(\text { tet }^{\mathrm{r}}\right)\right]\end{array}$ & Stratagene \\
\hline
\end{tabular}


Generation of plasmids, mutagenesis, and oligonucleotide primers for PCR. Plasmids were constructed using established methods (Sambrook et al., 1989). Plasmid transfer to bacteria was performed according to the protocol of Mandel \& Higa (1970). For in vitro mutagenesis, the QuikChange Site-Directed Mutagenesis kit (Stratagene) was used according to the manufacturer's instructions. DNA fragments were amplified employing Taq polymerase following the instructions of the manufacturer (Segenetic). Oligonucleotide primers used for PCR are summarized in Table 2.
$\boldsymbol{\beta}$-Galactosidase assay. Liquid medium was inoculated 1:10 with an overnight culture of the respective strain. To determine the influence of growth conditions on the transcriptional activity, overnight cultures were grown under the same conditions as the culture to be investigated. The culture was grown at $37{ }^{\circ} \mathrm{C}$ with agitation to $\mathrm{OD}_{600} 0.3-0.7$. To investigate the possible influence of oxygen pressure, static cultures were compared with cultures grown with intense shaking $(10 \mathrm{ml}$ culture in a $100 \mathrm{ml}$ Erlenmeyer flask at 180 r.p.m.). The $\beta$-galactosidase assays were performed as described

Table 2. Oligonucleotides used as primer pairs

Sequences shown in upper-case type are homologous to aah or aidA sequences. Recognition sequences for restriction enzymes are underlined.

\begin{tabular}{|c|c|c|}
\hline Oligonucleotide & Sequence $\left(5^{\prime}-3^{\prime}\right)$ & $\begin{array}{c}\text { Restriction } \\
\text { enzyme }\end{array}$ \\
\hline \multicolumn{3}{|c|}{ Amplification of promoter region fragments } \\
\hline A-443 & gctctagAGCAACATGTTCATGGCC & $X b a \mathrm{I}$ \\
\hline A-319 & gctctagaCTTGCATGGCATCTTGTTG & $X b a \mathrm{I}$ \\
\hline A-230 & gctctagaGATAACGTCAGATCTCATC & $X b a \mathrm{I}$ \\
\hline A-133 & gctctagaTATTGGCACATGGTATTATCC & $X b a \mathrm{I}$ \\
\hline $\mathrm{A}+114$ & tcggatccGTTAACGTGCCATTCTCC & BamHI, Нpa \\
\hline B-390 & gctctagATCATATTCCATGGGGAGC & $X b a \mathrm{I}$ \\
\hline B-334 & gctctagaGTAGATTTATTACGACATGCC & $X b a \mathrm{I}$ \\
\hline B-271 & gctctagATGGGCAAGTAGAATACCC & $X b a \mathrm{I}$ \\
\hline B-185 & gctctagACATGGCTGTAATGGATGC & $X b a \mathrm{I}$ \\
\hline IB-galK & aggtaCCTCGCGCGTTTCGG & $K p n \mathrm{I}$ \\
\hline B-317 & aggatccGCCAGTTTTTTTGTTGG & $K p n \mathrm{I}$ \\
\hline B-290 & aggatccGGATTGTCATGGCTG & KpnI \\
\hline B-118 & aggtaCCCTGTACACAAAGG & $K p n \mathrm{I}$ \\
\hline B-97 & aggatccGACAGTTTGAATGTACC & KpnI \\
\hline B-80 & aggtaCCGGCTTATTACAGGG & KpnI \\
\hline B-58 & aggatccTGTGGTGTGATTAGAAC & $K p n \mathrm{I}$ \\
\hline \multicolumn{3}{|c|}{ Site-directed mutagenesis } \\
\hline mutlfor & CGTCAGATCTCATCTcTgCAGAAAAAGATATC & PstI \\
\hline mut1rev & GATATCTTTTTCTGcAgAGATGAGATCTGACG & Pst I \\
\hline mut2for & GATCTCATCTTTTCAGcAgctGATATCTTTTATTCGCCAC & PvuII \\
\hline mut2rev & GTGGCGAATAAAAGATATCagcTgCTGAAAAGATGAGATC & PvuII \\
\hline aahfor & CTGGGTGACATTATTGCTTGG & \\
\hline aahrev & TTTGCTTGTGCGGTA & \\
\hline aidAfor & GGTGGCATTGCATTCCGG & \\
\hline aidArev & CCGGAAACATTGACCATACC & \\
\hline \multicolumn{3}{|l|}{$5^{\prime}$-RACE } \\
\hline lacZ & CAAGGCGATTAAGTTGG & \\
\hline$B+571$ & TCGAGGCAACGCCACCACTACTGACAC & \\
\hline polyC & СССССССССССССССССССС & \\
\hline
\end{tabular}


by Miller (1972). Enzyme activities were expressed as arbitrary Miller units and were derived from at least three independent experiments, each performed in triplicate.

RNA isolation and cDNA synthesis. RNA was isolated from E. coli strains with the RNeasy kit (Qiagen) following the addition of RNAprotect (Qiagen). To eliminate remaining plasmid and chromosomal DNA the Turbo DNA-free kit (Ambion) was used. Transcription from RNA to cDNA was performed with the RevertAid H Minus First Strand cDNA Synthesis kit (Fermentas). Random hexamers were used as primers.

Northern blotting. RNA molecules were separated by electrophoresis in agarose gels containing $2 \%$ formaldehyde, followed by incubation in $50 \mathrm{mM} \mathrm{NaOH}$ for $20 \mathrm{~min}$ to denature the RNA and a further $20 \mathrm{~min}$ in $10 \times \operatorname{SSC}(1.5 \mathrm{M} \mathrm{NaCl}, 0.15 \mathrm{M}$ sodium citrate, $\mathrm{pH}$ 7.0) for neutralization. The RNA was then transferred to a nylon membrane. After blotting, the membrane was incubated with $2 \times$ SSC for $5 \mathrm{~min}$ and dried, and the RNA was cross-linked to the membrane by UV radiation (Stratalinker, Stratagene). Hybridization was performed with the ECL Direct Nucleic Acid Labeling and Detection System (Amersham Bioscience). As a molecular mass standard, the RiboRuler High Range RNA ladder (Fermentas) was used. The RNA was visualized after ethidium bromide staining in a Lumi-Imager (Roche).

Rapid amplification of $5^{\prime}$ complementary DNA ends (5'-RACE). 5'-RACE was carried out according to Frohman (1993). RNA was isolated as described above. cDNA synthesis was performed with Avian Myeloblastosis Virus (AMV) reverse transcriptase (Promega). Total RNA $(5 \mu \mathrm{g})$ was incubated with $20 \mathrm{pmol}$ of specific oligonucleotide primers 1 in a total volume of $13.5 \mu \mathrm{l}$ at $65{ }^{\circ} \mathrm{C}$ for $5 \mathrm{~min}$. After $5 \mathrm{~min}$ on ice, $4 \mu \mathrm{l} \mathrm{AMV} \mathrm{RT-buffer,} 2 \mu 10 \mathrm{mM}$ dNTP mix and $5 \mathrm{U}$ AMV reverse transcriptase were added. After incubation at $50{ }^{\circ} \mathrm{C}$ for $1 \mathrm{~h}$, the enzyme was inactivated at $85{ }^{\circ} \mathrm{C}$ for $5 \mathrm{~min}$. The tailing reaction was performed with terminal transferase (BioLabs) and dGTP according to the manufacturer's instructions. The tailed cDNA was amplified by PCR using a polyC oligomer and a gene-specific primer 2. Primers and templates used are summarized in Table 3. PCR products were verified by sequencing (Seqlab, Göttingen).

Table 3. Primers used to determine the transcription start site

cDNA was synthesized from total RNA with specific primers (primer 1). After tailing of the cDNA, the DNA was further amplified with a gene-specific nested primer (primer 2) and a polyC oligonucleotide. The primers were chosen in accordance with the gene to be investigated and the plasmid of the recombinant strain used for RNA isolation.

\begin{tabular}{|lll|}
\hline Gene & Plasmid & \multicolumn{1}{c|}{ Primer } \\
\hline aah & pIB264 & Primer 1 aahrev \\
aah & pIB264 & Primer 2 A +114 \\
aah & pTvA515 & Primer 1 lacZ \\
aah & pTvA515 & Primer 2 A+114 \\
aidA & pIB264 & Primer 1 B +571 \\
aidA & pIB264 & Primer 2 B +85 \\
aidA & pMW334 & Primer 1 lacZ \\
aidA & pMW334 & Primer 2 B +85 \\
\hline
\end{tabular}

\section{RESULTS}

\section{Identification of aah and aidA promoter regions}

For stability and/or activity of AIDA-I as an adhesin, both the $a a h$ and the aidA genes need to be functional (Benz \& Schmidt, 2001). Recombinant E. coli strains harbouring aah and aidA genes either on the same (pIB264) or on different plasmids exhibit the same phenotype as the wildtype E. coli 2787 (Benz \& Schmidt, 1989, 2001). This indicates that both genes are transcribed from their own promoters. To identify the promoter regions of the two genes, transcriptional fusions of DNA fragments of varying lengths upstream of the aah or aidA start codon with the lac $Z$ coding region in the promoter test vector pCB192 were tested for activity. The various fragments included the upstream region (133-515 nt) and $114 \mathrm{nt}$ of the coding region of aah, and 58-390 nt of the upstream region and $85 \mathrm{nt}$ of the coding region of aidA. Immediately upstream of the lac $Z$ coding regions are stop codons in all three reading frames, so that we can exclude the synthesis of a fusion protein. Some of the plasmids harbouring small upstream regions were amplified by inverse PCR. In addition, we also obtained a construct containing $218 \mathrm{nt}$ of the upstream region of aah.

To monitor transcription, $\beta$-galactosidase activities of transcriptional fusion constructs were determined in the E. coli laboratory strain $\mathrm{DH} 5 \alpha$. For both aah and aidA genes, a correlation of the length of the upstream region to the $\beta$-galactosidase activity was observed. For aah, $218 \mathrm{nt}$ upstream of the start codon were found to be necessary for optimal transcription. Upstream of the start codon of aidA, 290 nt were needed for efficient mRNA synthesis (Fig. 1). Within these regions the ribosome-binding site, the transcription start site, the promoter region, and possibly also binding sites for putative regulatory proteins, were located. The transcription activities of the two promoter regions in the $\beta$-galactosidase assays differed by a factor of approximately three.

\section{Determination of transcription start sites}

To identify the transcription start sites, $5^{\prime}$-RACE experiments were performed. Total RNA from different recombinant $\mathrm{DH} 5 \alpha$ strains was isolated and reverse-transcribed to cDNA. Amplification of the $5^{\prime}$ fragment of the mRNA was done with polyG-tailed cDNA as template, polyC oligonucleotides, and a gene-specific primer 2 (Table 3 ). The polyG fragments were sequenced and the transcription start sites determined. For the aah gene, two transcription start sites, namely 128 and 149 nt upstream of the start codon, were identified. This was independent of whether the RNA was isolated from DH5 $\alpha$ harbouring pIB264 or the promoter test vector paah515. Sequences representing the -10 and -35 regions were located upstream of both sites (Fig. 2). For the start site at $128 \mathrm{nt}$, part of a UP element could also be detected. For the aidA gene also, two start sites were identified at 66 and 252 nt upstream of the 


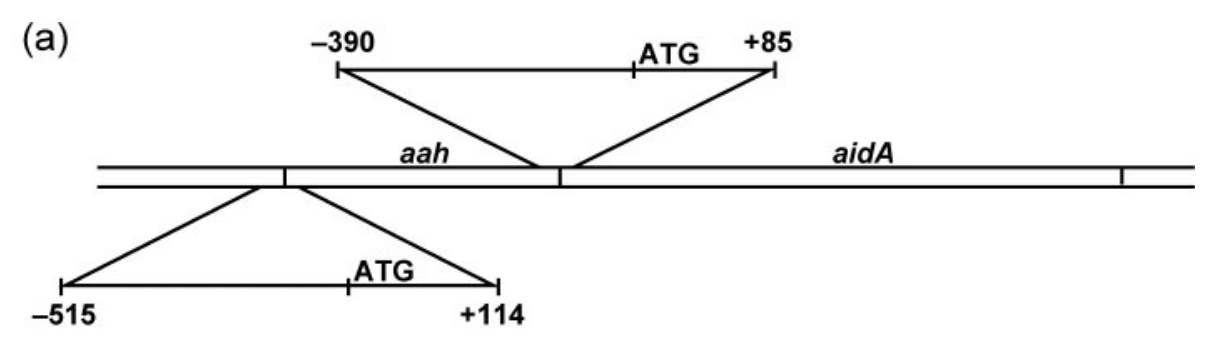

(b)

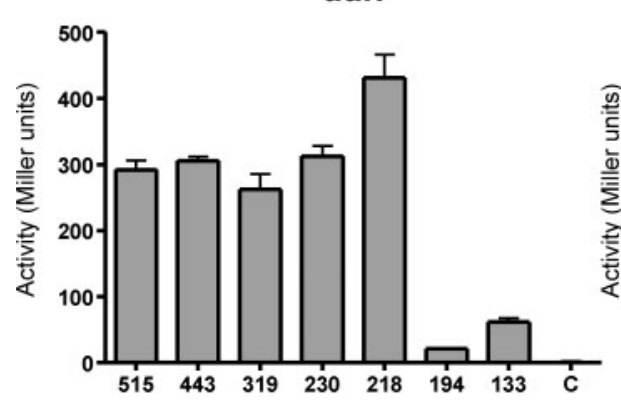

$\operatorname{aid} A$

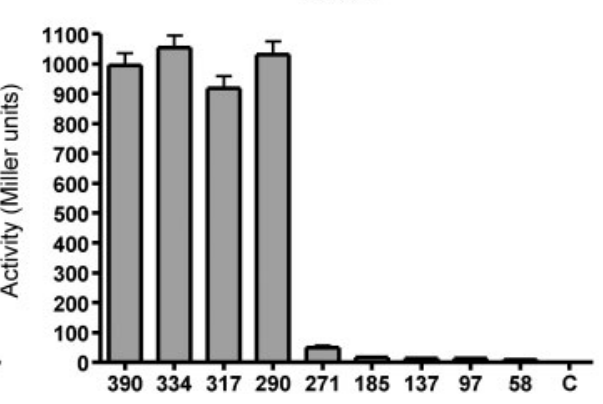

Fig. 1. Localization of the promoter region. (a) Schematic view of inserts containing a constant part of the coding region of either aah or aid $A$ and upstream regions of various lengths. (b) $\beta$-Galactosidase activity of recombinant $E$. coli $\mathrm{DH} 5 \alpha$ with a promoter test vector harbouring inserts containing part of the coding region of either aah (left) or aid $A$ (right) and upstream regions of various lengths. On the $x$ axis, the number of nucleotides upstream of the start codon is indicated. As a negative control, the vector pCB192 was used (C). Mean enzymatic activities are shown from at least three independent experiments performed in triplicate; error bars, SD.

start codon in DH5 $\alpha /$ pIB264. In the case of DH5 $\alpha /$ paidA334, only the start site at position 252 could be identified. Upstream of both sites, sequences representing the -10 and -35 motifs were identified (Fig. 2).

\section{Evidence for a bicistronic mRNA encompassing aah and aidA transcripts}

To obtain a functional AIDA-I adhesin, both gene products have to be present and functional in the appropriate ratio, as a low amount of the AAH enzyme might be sufficient to modify the AIDA protein. As both genes carry promoter sequences upstream of their start codons we were interested to see whether a basal level of both proteins could be synthesized via a bicistronic mRNA. To provide evidence for the additional presence of a bicistronic message, PCR and Northern blot analysis were performed.

RNA was isolated from E. coli DH5 $\alpha /$ pIB264 and transcribed into CDNA, and a multiplex PCR was performed with total cDNA as template. Plasmid pIB264 harbours the complete coding sequences for both genes, aah and aidA. The primers used lead to the amplification of an internal fragment for both aah (370 bp) and aidA (452 bp) (Niewerth et al., 2001) and, in addition, in the case of the presence of a bicistronic mRNA, to a fragment comprising the $3^{\prime}$ end of aah and the $5^{\prime}$ end of aidA (576 bp). As shown in Fig. 3(a), all three expected fragments were amplified as expected, thus providing evidence for a bicistronic mRNA.

To confirm these results, total RNA isolated from E. coli C600/pIB264 was separated by electrophoresis in a formaldehyde gel and subsequently blotted to a nylon membrane. Hybridization was performed with an aah-specific probe. RiboRuler High Range RNA was used as a size standard. As shown in Fig. 3(b), aah-specific mRNA with a size of about $5300 \mathrm{nt}$ could be detected. Transcription from the aah promoter apparently leads to a bicistronic mRNA comprising aah (1173 nt) and aidA (3858 nt).

\section{Influence of growth conditions on transcription activity}

Experiments to identify the minimal regions necessary for transcription of the two genes were performed with bacteria grown in rich medium. To investigate the expression of aah and aidA under different conditions, bacteria were also grown in minimal medium, at different temperatures and oxygen pressures, and were further analysed in different growth phases. To ensure that no regulatory region(s) might be deleted, the experiments were performed with DH5 $\alpha$ l paah515 and DH5 $/$ /paidA334. Expression of the aah and aidA genes was found to be highly susceptible to variations in growth conditions (Fig. 4). By monitoring expression 


\section{aah}

\begin{tabular}{|c|c|}
\hline 200 & $\begin{array}{cc}\mathrm{CH}_{3} & \text { Inverted repeat region } \\
\text { AGATATCTTTTATTCGCCACGACACAAGAAAAAAAAACTATTATTAAGAA }\end{array}$ \\
\hline & 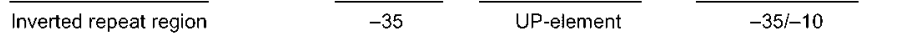 \\
\hline-150 & $\begin{array}{l}\text { TGTATTTTGATTATTACTATTGGCACATGGTATTATCCAGAAAATTACAA } \\
\text { TSS149 }\end{array}$ \\
\hline 100 & АСАTATATTAAACTCTTTCTTTTAAGAATTTATTATATCCGTTCTAGTTA \\
\hline & $\begin{array}{c}\text { TCTTGGATATTACATTATAATATTGGATATTACATTATATAGGAGGGATT } \\
\text { Direct repeat }\end{array}$ \\
\hline & ATGACTTTCTTATCACCACCAGAGATACCAACAATAAAAGCAGATAATGC \\
\hline
\end{tabular}

aidA
Fig. 2. Sequences upstream of the start codons of aah and aidA. The figure shows the nucleotide sequences of the promoter regions of aah and aidA. The positions of the transcription start sites (TSS) and the -10 and -35 regions are indicated. The start codon and the ribosome-binding sites (RBS) are also marked. from the promoter region upstream of aah, high $\beta$ galactosidase activity was measured at $37{ }^{\circ} \mathrm{C}$, at low oxygen, in salt medium and in stationary phase. The most prominent (a)

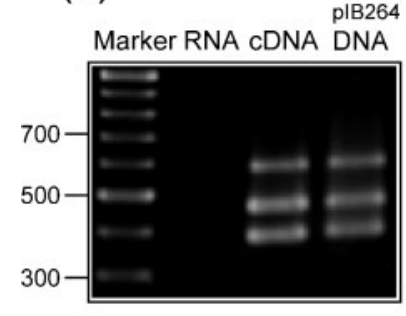

(b)

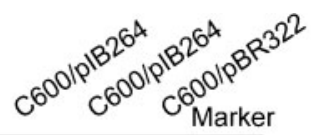

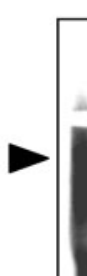

difference was seen for temperature dependence (Fig. 4). Interestingly, expression under the control of the aidA promoter was higher under high oxygen than under low oxygen pressure. However, a combination of 'enhancing conditions' to further increase expression of AIDA-I did not result in an additive effect (data not shown).

\section{Influence of the specific background of the $E$. coli expression strain on the transcription activity of aah and aidA promoters}

In the expression of recombinant proteins with the help of the AIDA system, the genetic background of the particular E. coli strain used might additionally influence transcription activity and expression. To assess the effect of various genotypes on transcription, the activity of the aah and aidA promoters was investigated in several E. coli K-12 laboratory strains that are often employed for protein expression experiments. Not only the expression of lac $Z$ from the aah or aidA promoter varied in the different strains but, moreover, we also observed a remarkable difference in activity between the two promoters in the same strain. Not even very closely related strains, such as DH5 $\alpha$, JM109, Sure and XL1 blue, as derivatives of the E. coli strain DH1, or strains BW25113 and its derivative BW25141, exhibited similar expression patterns (Fig. 5).

\section{Influence of rpos, hns and rfaH on the transcription of the aah and aidA genes}

Optimal expression under the control of the aah promoter was seen in stationary phase, in low oxygen and during 
(a)

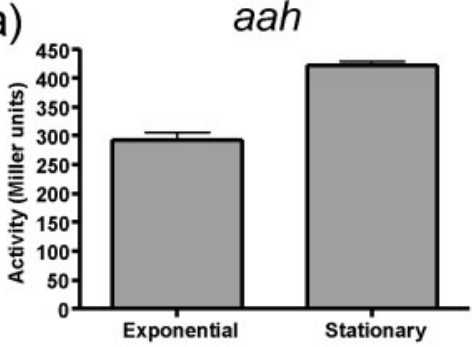

(b)

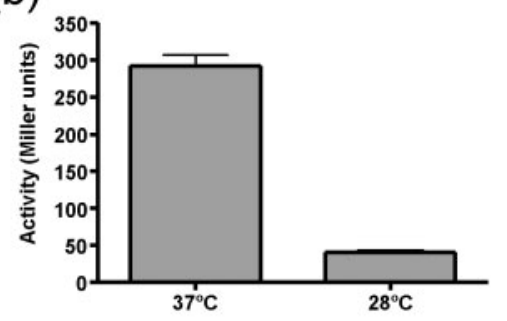

(c)

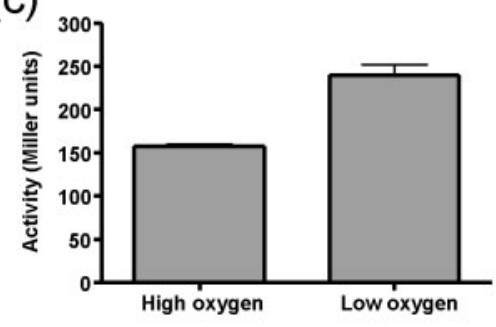

(d)

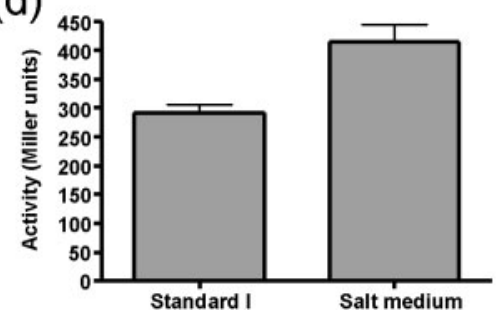

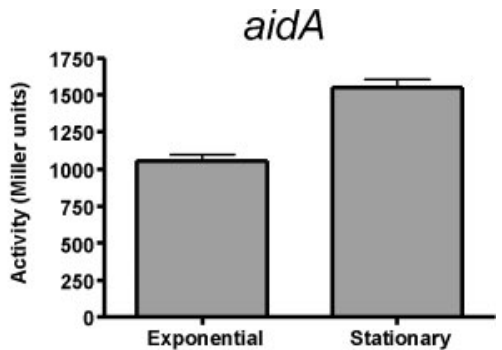
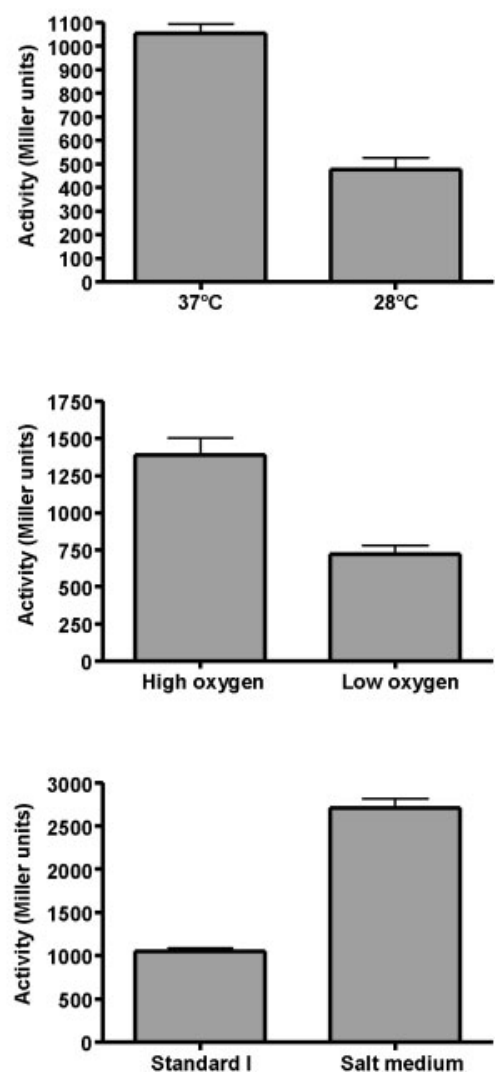

Fig. 4. Influence of growth conditions on transcription activity. $\beta$-Galactosidase activity of recombinant $E$. coli $\mathrm{DH} 5 \alpha$ with a promoter test vector harbouring $515 \mathrm{nt}$ upstream of the start codon of aah or $334 \mathrm{nt}$ upstream of the start codon of aidA. The bacteria were grown at different growth phases (a), at different temperatures (b), in different oxygen conditions (c) and in different media (d). Mean enzymatic activities are shown from at least three independent experiments performed in triplicate; error bars, SD. growth in salt medium, which indicated that the sigma factor RpoS might be involved in transcription. To investigate this further, we transformed $E$. coli strain RH90, an E. coli rpoS mutant, with the promoter test vector pCB192 containing 515 nt upstream of aah, and subsequently assessed the expression of the lac $Z$ gene. We found a significant reduction in transcription activity in the $r p o S$ mutant compared to the wild-type isogenic strain MC4100 (Fig. 6a). Interestingly, transcription from the aidA promoter appeared not to be influenced by RpoS.

Another global regulator is the histone-like protein $\mathrm{H}-\mathrm{NS}$ (e.g. Dorman, 2004). The influence of this protein on the expression from aah and aidA promoters was determined in an hns mutant (Dersch et al., 1994). In the absence of $\mathrm{H}$ NS, both promoters showed reduced activity (Fig. 6b). Although H-NS has been reported in most cases to exert a repressor function (Fang \& Rimsky, 2008), the AIDA system seems to be one of the exceptions where H-NS appears to act as an activator. At present, however, it cannot be determined whether H-NS functions directly or, alternatively, indirectly influences expression of the aah and aidA genes.

In addition, based on reports that $r f a H$ influences biofilm formation by modulating expression of the abundant $E$. coli autotransporter Ag43 (Beloin et al., 2006; Van der Woude \& Henderson, 2008), we were interested to investigate the effect of $r f a H$ on the expression of aah and aidA (Fig. 6c). Interestingly, in $r f a H$ mutants, expression of aah and of aidA was enhanced as compared with wild-type bacteria.

\section{Influence of upstream repeat motifs on transcriptional activity}

An inverted repeat region has been identified at the extreme $5^{\prime}$ end of the fragment upstream of the aah start 

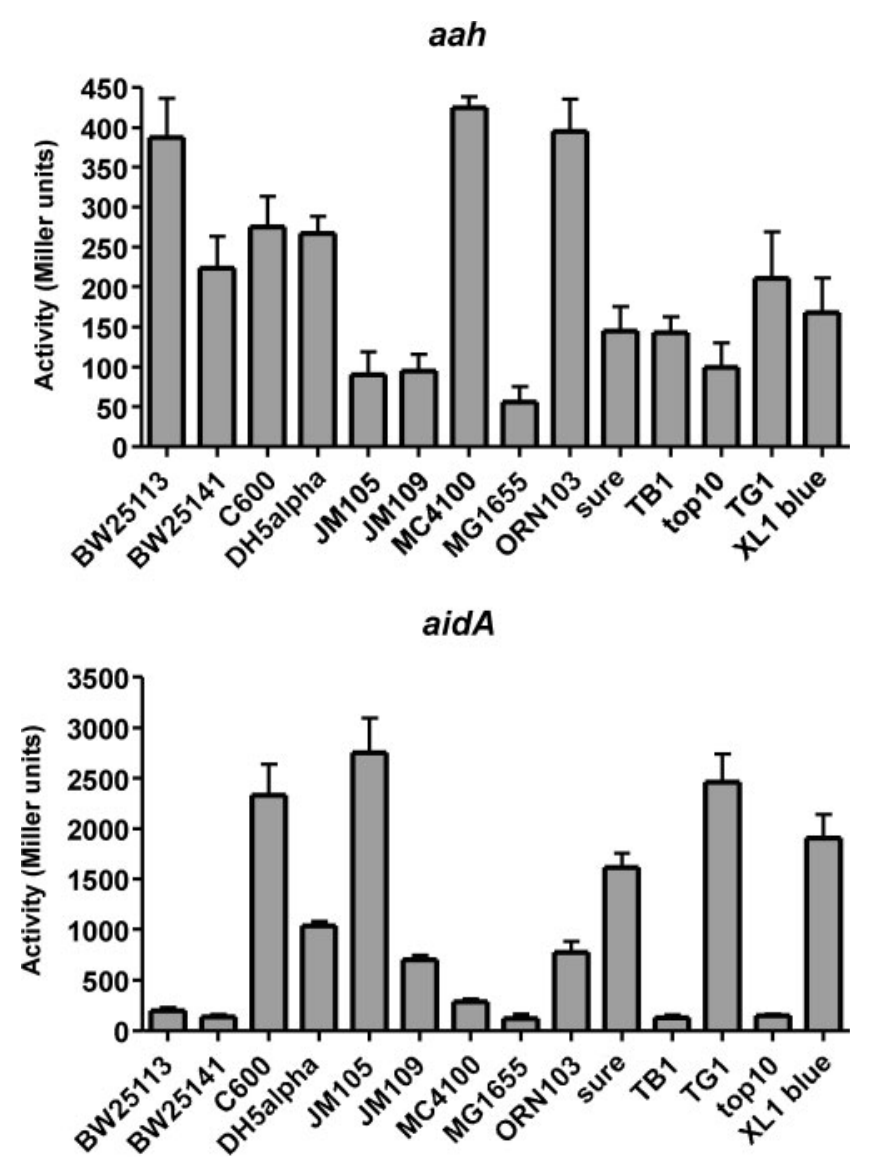

Fig. 5. Influence of $E$. coli strain on transcription activity. $\beta$ Galactosidase activities of recombinant $E$. coli strains with either a promoter test vector harbouring $515 \mathrm{nt}$ upstream of the start codon of aah or $334 \mathrm{nt}$ upstream of the start codon of aidA. Mean enzymatic activities are shown from at least three independent experiments performed in triplicate; error bars, SD.

codon mediating maximum transcription rates. This region exhibited two identical motifs and one motif harbouring an inverted nucleotide sequence in the middle (Fig. 7), constituting two different inverted repeats. Deletion of this region resulted in a remarkable decrease in activity (Fig. 1). To examine whether the inverted repeats might be important for transcription, site-directed mutagenesis was performed. Here, either a single motif was altered or the two identical motifs were mutated in such a way that at least one of the inverted repeats was destroyed. Every mutation introduced showed reduced transcription rates (Fig. 7). Moreover, a mutation in the second motif leading to the loss of the inverted repeat sequence also resulted in a nearly complete loss of promoter activity.

\section{DISCUSSION}

The products of the aah and aidA genes, AAH and AIDA-I, constitute the AIDA system, which mediates the diffuse
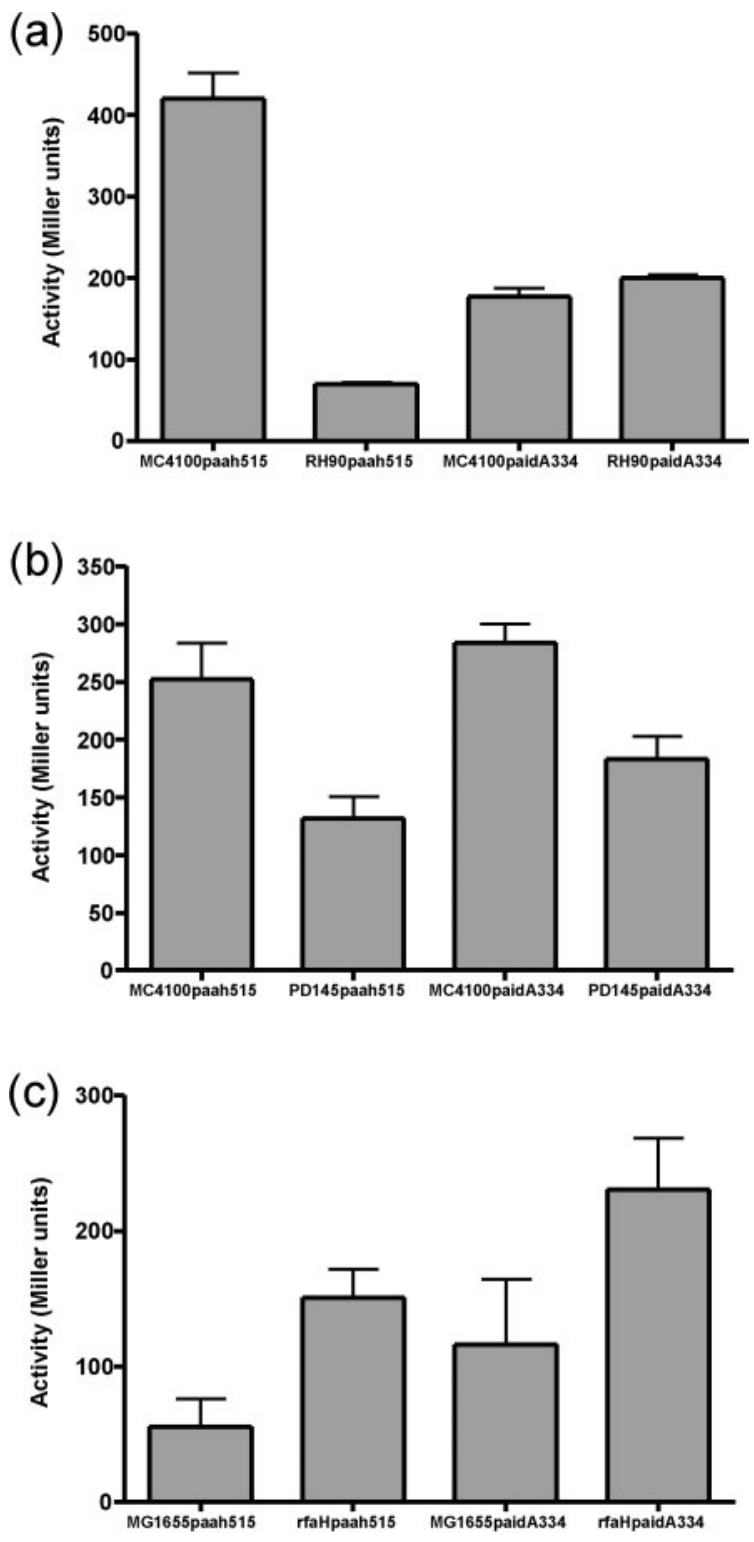

Fig. 6. Influence of RpoS, H-NS and RfaH on transcription activity. $\beta$-Galactosidase activities of recombinant $E$. coli strains with either a promoter test vector harbouring $515 \mathrm{nt}$ upstream of the start codon of aah or $334 \mathrm{nt}$ upstream of the start codon of aidA were determined. The E. coli strains were rpoS (a), hns (b) (both in a MC4100 background) or $\mathrm{rfaH}$ (c) mutants in a MG1655 background. The MC4100 wild-type strains were derived from different laboratories and were compared with the appropriate mutant strain. Mean enzymatic activities are shown from at least three independent experiments performed in triplicate; error bars, SD.

adherence phenotype of E. coli 2787 to HeLa cells. For full activity it is imperative that both proteins are present and functional (Benz \& Schmidt, 2001). In this study, we investigated the promoter organization and transcription of the two genes to gain further insight into their regulation. 
(a)

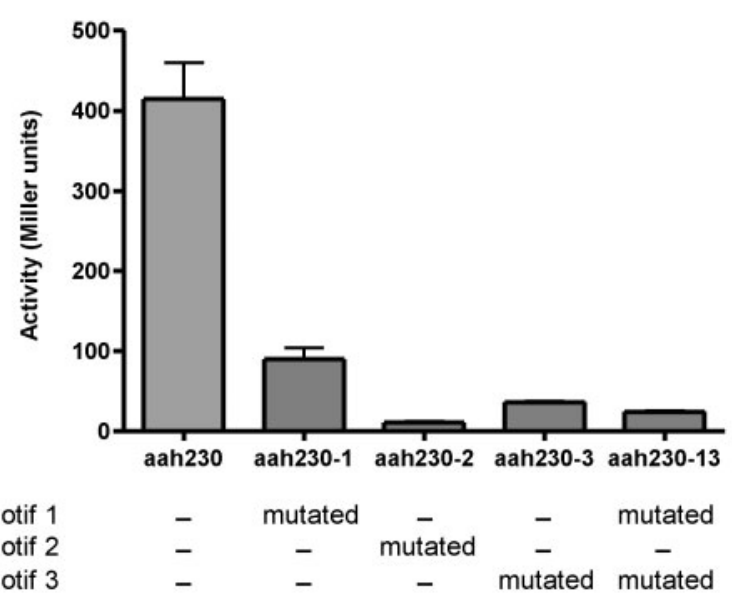

(b)

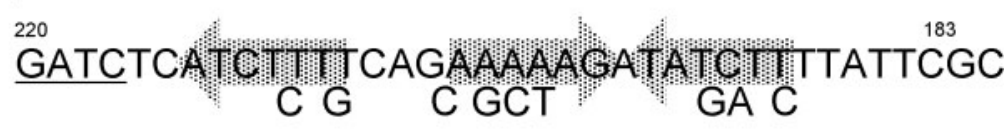

Motif $1 \quad$ Motif $2 \quad$ Motif 3

Fig. 7. Influence of the inverted repeat region on transcription activity. (a) $\beta$-Galactosidase activity of recombinant $E$. coli $\mathrm{DH} 5 \alpha$ with a promoter test vector harbouring $230 \mathrm{nt}$ upstream of the start codon of aah was determined and compared with that of strains harbouring plasmids with point mutations within this region. Mean enzymatic activities are shown from at least three independent experiments performed in triplicate; error bars, SD. (b) Sequence of the repeat region; locations of the mutations are indicated. The GATC motif for methylation is underlined.
Previous studies from our laboratory have indicated that $220 \mathrm{nt}$ upstream of the start codon of aah (Benz \& Schmidt, 2001) and 384 nt upstream of aidA (unpublished results) are sufficient for synthesis of the functional AAH and AIDA-I proteins. To further characterize the promoter region(s) and to identify possible regulator sites, deletion mutants of the upstream regions of aah and aidA were created and 5'-RACE experiments were performed. For both genes, the transcription start sites were identified, the promoter regions were localized, and additional insight concerning regulatory structures was obtained.

The transcription of the aah gene starts at two different sites. For both start sites, -10 and -35 regions were identified. Comparison of the sequences upstream of the start sites with the consensus sequences of $\sigma^{70}$ and $\sigma^{\mathrm{S}}$ promoters (Table 4) showed a high homology to both of the promoters at position 149 and only homology to the
-10 region for the start site at position 128 . It is known that both factors can bind to the same promoter, albeit with different efficiencies. Transcription activity in a $r p o S$ negative $E$. coli strain was markedly reduced, indicating that $\sigma^{\mathrm{S}}$ apparently takes part in transcription initiation. It has been demonstrated that this alternative sigma factor can also bind to a degenerate -35 region (Typas et al., 2007).

Besides the binding sites for the RNA polymerase there are two regions located upstream of aah that might be involved in regulation. One region contains three sequence motifs that would be sufficient for the formation of two inverted repeats. As sequences with dyad symmetry in the neighbourhood of a promoter are known to be binding sites for regulatory proteins this might also be the case for the aah promoter. Mutations in this region led to a dramatic decrease in transcription activity that clearly

Table 4. Comparison of the sequence of the upstream region of aah with the consensus sequences of $\sigma^{70}$ and $\sigma^{S}$ promoter regions according to Typas et al. (2007)

\begin{tabular}{|c|c|}
\hline Promoter region & Sequence \\
\hline \multicolumn{2}{|c|}{ Promoter region upstream of position -149} \\
\hline aah sequence & AAAAGATATCTTTTATTCGCCACGACACAAGAAAAAAAAACTATTATTAAGAATG \\
\hline$\sigma^{70}$ consensus & AAWWWTWTTTTNNAAAA—TTGACA- — TRTGNTATAAT—R \\
\hline$\sigma^{\mathrm{S}}$ consensus & AAAWWWWTTTT— \\
\hline \multicolumn{2}{|c|}{ Promoter region upstream of position -128} \\
\hline aah sequence & CACGACACAAGAAAAAAAAACTATTATTAAGAATGTATTTTGATTATTACTATTGG \\
\hline$\sigma^{70}$ consensus & AAWWWTWTTTTNNAAAA—TTGACA-TRTGNTATAAT—R \\
\hline$\sigma^{\mathrm{S}}$ consensus & AAAWWWWTTTT— \\
\hline
\end{tabular}


indicated an important role for this motif in transcription regulation. Interestingly, in a database search we could not identify sequence homologies to known regulator-binding sites.

Further, we wondered whether the AT-rich region might harbour structural elements that would allow the binding of additional regulatory factors. A protein that is known to bind to bent DNA is the histone-like protein H-NS. Normally, H-NS serves as a negative regulator (Fang \& Rimsky, 2008), and in hns-negative strains transcription activity is usually increased as compared with the wild-type strain. In some cases, such as for the csiD promoter of $E$. coli, H-NS has also been reported to actually activate transcription (Germer et al., 2001). However, for csiD, as for aah and aidA, conclusions about whether H-NS exerts its positive effect directly or indirectly cannot be drawn.

The influence of $\mathrm{RfaH}$ on transcription of aah and aidA was found to be opposite to the usually observed effect of this regulator. $\mathrm{RfaH}$ is described as a transcriptional antiterminator interacting with the elongation complex of the RNA polymerase for efficient transcription of polycistronic mRNAs (Xue et al., 2009). Normally, interaction of RfaH with the RNA polymerase depends on the ops element located in the $5^{\prime}$ untranslated region (UTR) of the mRNA (Artsimovitch \& Landick, 2002). This ops element cannot be detected in the 5' UTR of aah and aidA mRNA. Nevertheless, there was an increase in transcription activity for both genes in an $\mathrm{rfaH}$ mutant. Similar results have been reported for the related E. coli autotransporter Ag43 encoded by the $f l u$ gene (Beloin et al., 2006). The 5' UTR of the $f l u$ mRNA also shows no homology to the ops element; however, increased transcription of the gene in an $r f a H$ mutant has been found. As in the case of H-NS, the effect of $\mathrm{RfaH}$ on aah and aidA transcription might be indirect.

Initiation of transcription from the aah promoter(s) leads to the synthesis of a bicistronic mRNA for aah and aidA. Both genes have to be expressed to mediate and/or stabilize the adherence function of AIDA-I. As AAH is an enzyme it does not need to be expressed in equimolar amounts. Therefore, a possibility to increase the amount of the AIDA-I adhesin might be via the synthesis of additional aidA mRNA, utilizing the specific aidA promoter.

Investigating the upstream region of aidA by $5^{\prime}$-RACE we identified two distinct transcription start sites. Both sites have -10 and -35 regions with high similarity to the consensus sequence of a $\sigma^{70}$ promoter, and this is further supported by the distance between the two regions (Typas et al., 2007). Interestingly, the start site at $66 \mathrm{nt}$ upstream of the start codon could only be identified in the strain harbouring a plasmid with the complete coding sequence for aah and aidA, and not in the promoter test vector harbouring only $85 \mathrm{nt}$ encoding the $\mathrm{N}$-terminal sequence. This result points to an additional influence of the downstream sequence and/or the gene products on expression. The transcription start site at $252 \mathrm{nt}$ could be identified in both recombinant strains.
Gene regulation in micro-organisms is influenced by various environmental factors, and pathogenic micro-organisms are no exception (Finlay \& Falkow, 1997). Even if specific regulators involved in transcription of aah and aidA have not been identified up to now, differences in expression were detected depending on growth phase, nutrition, oxygen and, particularly, temperature. The involvement of $\sigma^{\mathrm{S}}$ in the transcription of aah could be verified by experiments performed in an rpoS-negative strain. $\sigma^{\mathrm{S}}$ is a stationary phase-specific sigma factor and functions as a global regulator of gene expression under various stress conditions (Klauck et al., 2007). Transcription of aah by a $\sigma^{\mathrm{S}}$ RNA polymerase fits quite well with the environment normally colonized by enteropathogenic E. coli, and such transcription is often modulated further by additional regulators and histone-like proteins (Germer et al., 2001). The changes found in the transcription activity of aidA could not be referred to one single factor.

Interestingly, we observed a difference in transcription activity in E. coli MC4100 strains derived from different laboratory stocks. Such differences in transcription activity have also been reported by other investigators (Jishage \& Ishihama, 1997; Soupene et al., 2003), as bacteria from a single origin are subject to genome plasticity and, therefore, exhibit frequent changes, resulting in laboratory-specific alterations and characteristics. Much greater differences in activity have been detected by comparing different E. coli $\mathrm{K}-12$ strains. This might be of practical interest, as the AIDA system is employed by numerous groups for surface expression studies with heterologous antigens. Not only did we measure different transcription activities for $a a h$ (by a factor of five) and aidA (by a factor of 20), but also the ratio between the two genes differed by a factor of approximately 60 . This further supports the finding that the transcription of aah and aidA is modulated in different ways. In view of the known genotypes of the different $E$. coli strains examined in this study, a common reason for these differences cannot be given. The observed differences could be due to a polymorphism(s) in a varying number of genes. Spira et al. (2008) have shown strain variations in ppGpp concentration and $\sigma^{\mathrm{S}}$ level in laboratory strains of E. coli K-12 due to mutations in spoT and rpoS. This kind of microevolution might also take place in other genes and influence global bacterial regulatory networks. To study the regulation of the AIDA system not only with respect to the two genes of the AIDA autotransporter system but also in the context of pathogenicity it will be interesting to investigate expression in the wild-type strain E. coli 2787.

Up to now, a correlation between transcription of the aah and aidA genes and protein synthesis has not been experimentally proven. However, there is a first hint that the amount of mRNA correlates with the amount of protein detectable. Berthiaume et al. (2007) attempted to isolate AIDA-I from the recombinant E. coli strain ED9 and failed. Strain ED9 is an MC4100 derivative, and in transcription assays it has been shown that in MC4100 only a very low activity can be detected for aidA, whereas in 
strain C600, which is normally used to isolate usable amounts of AIDA-I, the activity of the aidA promoter is eightfold higher.

In conclusion, this study shows that both genes of the AIDA system are transcribed as a bicistronic mRNA. There is additional transcription from the aidA promoter, increasing the amount of mRNA. However, this does not reflect the differences at the protein level seen in the bacterium. Therefore, to explain the observed differences at the protein level there have to be other, additional factors involved, such as mRNA stability, translation efficiency and/or half-life of the specific gene products. Not surprisingly, transcription varied considerably in different E. coli K-12 laboratory strains and under different environmental conditions. Interestingly, transcription of both genes was enhanced in $h n s$ and $r f a H$ mutant strains, a finding that needs to be investigated in future studies.

\section{ACKNOWLEDGEMENTS}

We are indebted to P. Dersch, U. Dobrindt, R. Hengge and G. Heusipp for providing mutant $E$. coli strains. This study was supported in part by grants from the Deutsche Forschungsgemeinschaft (DFG SFB 293 TP B5) and by a grant from the EU Network ERA-NET PathoGenoMics (project no. 03113937C).

\section{REFERENCES}

Appleyard, R. K. (1954). Segregation of new lysogenic types during growth of a doubly lysogenic strain derived from Escherichia coli K12. Genetics 39, 440-452.

Artsimovitch, I. \& Landick, R. (2002). The transcriptional regulator $\mathrm{RfaH}$ stimulates RNA chain synthesis after recruitment to elongation complexes by the exposed nontemplate DNA strand. Cell 109, 193203.

Bäcklund, E., Markland, K. \& Larsson, G. (2008). Cell engineering of Escherichia coli allows high cell density accumulation without fedbatch process control. Bioprocess Biosyst Eng 31, 11-20.

Beloin, C., Michaelis, K., Lindner, K., Landini, P., Hacker, J., Ghigo, J.-M. \& Dobrindt, U. (2006). The transcriptional anti-terminator $\mathrm{RfaH}$ represses biofilm formation in Escherichia coli. J Bacteriol 188, 1316-1331.

Benz, I. \& Schmidt, M. A. (1989). Cloning and expression of an adhesin (AIDA-I) involved in diffuse adherence of enteropathogenic Escherichia coli. Infect Immun 57, 1506-1511.

Benz, I. \& Schmidt, M. A. (1992a). AIDA-I, the adhesin involved in diffuse adherence of the diarrhoeagenic Escherichia coli strain 2787 (O126: H27), is synthesized via a precursor molecule. Mol Microbiol 6, 1539-1546.

Benz, I. \& Schmidt, M. A. (1992b). Isolation and serologic characterization of AIDA-I, the adhesin mediating the diffuse adherence phenotype of the diarrhea-associated Escherichia coli strain 2787 (O126: H27). Infect Immun 60, 13-18.

Benz, I. \& Schmidt, M. A. (2001). Glycosylation with heptose residues mediated by the aah gene product is essential for adherence of the AIDA-I adhesin. Mol Microbiol 40, 1403-1413.

Berthiaume, F., Rutherford, N. \& Mourez, M. (2007). Mutations affecting the biogenesis of the AIDA-I autotransporter. Res Microbiol 158, 348-354.
Brandon, L. D., Goehring, N., Janakiraman, A., Yan, A. W., Wu, T., Beckwith, J. \& Goldberg, M. B. (2003). IcsA, a polarly localized autotransporter with an atypical signal peptide, uses the Sec apparatus for secretion, although the $\operatorname{Sec}$ apparatus is circumferentially distributed. Mol Microbiol 50, 45-60.

Casali, N., Konieczny, M., Schmidt, M. A. \& Riley, L. W. (2002). Invasion activity of a Mycobacterium tuberculosis peptide presented by the Escherichia coli AIDA autotransporter. Infect Immun 70, 68466852.

Datsenko, K. A. \& Wanner, B. L. (2000). One-step inactivation of chromosomal genes in Escherichia coli K-12 using PCR products. Proc Natl Acad Sci U S A 97, 6640-6645.

Dautin, N. \& Bernstein, H. D. (2007). Protein secretion in Gramnegative bacteria via the autotransporter pathway. Annu Rev Microbiol 61, 89-112.

Dersch, P., Kneip, S. \& Bremer, E. (1994). The nucleoid-associated DNA-binding protein H-NS is required for the efficient adaptation of Escherichia coli K-12 to a cold environment. Mol Gen Genet 245, 255259.

Dorman, C. J. (2004). H-NS: a universal regulator for a dynamic genome. Nat Rev Microbiol 2, 391-400.

Fang, F. C. \& Rimsky, S. (2008). New insights into transcriptional regulation by H-NS. Curr Opin Microbiol 11, 113-120.

Finlay, B. B. \& Falkow, S. (1997). Common themes in microbial pathogenicity revisited. Microbiol Mol Biol Rev 61, 136-169.

Frohman, M. A. (1993). Rapid amplification of complementary DNA ends for generation of full-length complementary DNAs: thermal RACE. Methods Enzymol 218, 340-356.

Germer, J., Becker, G., Metzner, M. \& Hengge-Aronis, R. (2001). Role of activator site position and a distal UP-element half-site for sigma factor selectivity at a CRP/H-NS-activated $\sigma^{\mathrm{S}}$-dependent promoter in Escherichia coli. Mol Microbiol 41, 705-716.

Gibson, T. J. (1984). Studies on the Epstein-Barr virus genome. $\mathrm{PhD}$ thesis, University of Cambridge.

Hanahan, D. (1983). Studies on transformation of Escherichia coli with plasmids. J Mol Biol 166, 557-580.

Henderson, I. R., Navarro-Garcia, F., Desvaux, M., Fernandez, R. C. \& Ala'aldeen, D. (2004). Type V protein secretion pathway: the autotransporter story. Microbiol Mol Biol Rev 68, 692-744.

Jain, S. \& Goldberg, M. B. (2007). Requirement for YaeT in the outer membrane assembly of autotransporter proteins. J Bacteriol 189, 5393-5398.

Jensen, K. F. (1993). The Escherichia coli K-12 "wild types" W3110 and MG1655 have an rph frameshift mutation that leads to pyrimidine starvation due to low pyrE expression levels. J Bacteriol 175, 3401-3407.

Jishage, M. \& Ishihama, A. (1997). Variation in RNA polymerase sigma subunit composition within different stocks of Escherichia coli W3110. J Bacteriol 179, 959-963.

Jose, J. \& Meyer, T. F. (2007). The autodisplay story: from discovery to biotechnical and biomedical applications. Microbiol Mol Biol Rev 71, 600-619.

Klauck, E., Typas, A. \& Hengge, R. (2007). The sigmaS subunit of RNA polymerase as a signal integrator and network master regulator in the general stress response in Escherichia coli. Sci Prog 90, 103127.

Konieczny, M. P. J., Suhr, M., Noll, A., Autenrieth, I. B. \& Schmidt, M. A. (2000). Cell surface presentation of recombinant (poly-)peptides including functional $\mathrm{T}$ cell epitopes by the AIDA autotransporter system. FEMS Immunol Med Microbiol 27, 321-332. 
Laarmann, S. \& Schmidt, M. A. (2003). The Escherichia coli AIDA autotransporter adhesin recognizes an integral membrane glycoprotein as receptor. Microbiology 149, 1871-1882.

Lange, R. \& Hengge-Aronis, R. (1991). Identification of a central regulator of stationary-phase gene expression in Escherichia coli. Mol Microbiol 5, 49-59.

Mainil, J. G., Jacquemin, E., Pohl, P., Kaeckenbeeck, A. \& Benz, I. (2002). DNA sequences coding for the F18 fimbriae and AIDA adhesin are localised on the same plasmid in Escherichia coli isolates from piglets. Vet Microbiol 86, 303-311.

Mandel, M. \& Higa, A. (1970). Calcium dependent bacteriophage DNA infection. J Mol Biol 53, 159-162.

Marinus, M. G., Carraway, M., Frey, A. Z., Brown, L. \& Arraj, J. A. (1983). Insertion mutations in the dam gene of Escherichia coli K-12. Mol Gen Genet 192, 288-289.

Miller, J. H. (1972). Experiments in Molecular Genetics. Cold Spring Harbor, NY: Cold Spring Harbor Laboratory.

Niewerth, U., Frey, A., Voss, T., Le Bouguénec, C., Baljer, G., Franke, S. \& Schmidt, M. A. (2001). The AIDA autotransporter system is associated with F18 and Stx2e in Escherichia coli isolates from pigs diagnosed with edema disease and postweaning diarrhea. Clin Diagn Lab Immunol 8, 143-149.

Orndorff, P. E., Spears, P. A., Schauer, D. \& Falkow, S. (1985). Two modes of control of pilA, the gene encoding type 1 pilin in Escherichia coli. J Bacteriol 164, 321-330.

Powers, E. L. \& Randall, L. L. (1995). Export of periplasmic galactosebinding protein in Escherichia coli depends on the chaperone SecB. J Bacteriol 177, 1906-1907.

Pritchard, J., Ngeleka, M. \& Middleton, D. M. (2004). In vivo and in vitro colonization patterns of AIDA-I-positive Escherichia coli isolates from piglets with diarrhea. J Vet Diagn Invest 16, 108115 .
Sambrook, J., Frisch, E. F. \& Maniatis, T. (1989). Molecular Cloning: $a$ Laboratory Manual, 2nd edn. Cold Spring Harbor, NY: Cold Spring Harbor Laboratory.

Schneider, K. \& Beck, C. F. (1986). Promoter-probe vectors for the analysis of divergently arranged promoters. Gene 42, 37-48.

Soupene, E., van Heeswijk, W. C., Plumbridge, J., Stewart, V., Bertenthal, D., Lee, H., Prasad, G., Paliy, O., Charernnoppakul, P. \& Kustu, S. (2003). Physiological studies of Escherichia coli strain MG1655: growth defects and apparent cross-regulation of gene expression. J Bacteriol 185, 5611-5626.

Spira, B., Hu, X. \& Ferenci, T. (2008). Strain variation in ppGpp concentration and RpoS levels in laboratory strains of Escherichia coli K-12. Microbiology 154, 2887-2895.

Suhr, M., Benz, I. \& Schmidt, M. A. (1996). Processing of the AIDA-I precursor: removal of $\operatorname{AIDA}^{\mathrm{c}}$ and evidence for the outer membrane anchoring as a $\beta$-barrel structure. Mol Microbiol 22, 31-42.

Typas, A., Becker, G. \& Hengge, R. (2007). The molecular basis of selective promoter activation by the $\sigma^{\mathrm{S}}$ subunit of RNA polymerase. Mol Microbiol 63, 1296-1306.

Van der Woude, M. W. \& Henderson, I. R. (2008). Regulation and function of Ag43 (Flu). Annu Rev Microbiol 62, 153-169.

Wells, T. J., Tree, J. J., Ulett, G. C. \& Schembri, M. A. (2007). Autotransporter proteins: novel targets at the bacterial surface. FEMS Microbiol Lett 274, 163-172.

Xue, P., Corbett, D., Goldrick, M., Naylor, C. \& Roberts, I. S. (2009). Regulation of expression of the region 3 promoter of the Escherichia coli K5 capsule gene cluster involves H-NS, SlyA, and a large 5' untranslated region. J Bacteriol 191, 1838-1846.

Yanisch-Perron, C., Vieira, J. \& Messing, J. (1985). Improved M13 phage cloning vectors and host strains: nucleotide sequences of the M13mpl8 and pUC19 vectors. Gene 33, 103-111.

Edited by: R. G. Sawers 